\title{
The Effects of Coupling Agents on the Mechanical and Thermal Properties of Eucalyptus Flour/HDPE Composite
}

\author{
Siripan Metanawin ${ }^{1, a}$, Phiromrat Pengrung ${ }^{1}$ and Tanapak Metanawin ${ }^{2}$ \\ ${ }^{1}$ Department of Textile Engineering, Faculty of Engineering, Rajamangala University of Technology Thanyaburi, PathumThani, 12110, \\ Thailand \\ ${ }^{2}$ Department of Materials and Production Technology Engineering, Faculty of Engineering, King Mongkut's University of Technology \\ North Bangkok, Bangkok, 10800, Thailand
}

\begin{abstract}
The aim of this research was to study the effects of the coupling agents, Fusabond ${ }^{\mathrm{TM}}$ E-528 (polyethylenegrafted maleic anhydride; PE-g-MA, MA) and Amino Silane (Si), on the thermal properties, and mechanical properties of Eucalyptus flour-HDPE composite. Variation of the Eucalyptus flour contents in the HDPE resulted in properties of the composite. With increasing in the contents of Eucalyptus flour in polymer matrix, the mechanical properties of the HDPE composite decreased in EU-MA series samples while they were gradually decreased in EU-Si series samples. SEM micrographs showed the fracture surface of the HDPE/Eucalyptus composite at different ratios of Eucalyptus flour. SEM micrograpgh exhibited the dispersion of EU flour in polymer matrix. The samples of both coupling agents showed an increase in interfacial adhesion, observed for the considerable decreased of gaps between the matrix and the dispersed phase. However, the EU-MA sample appeared to be more uniformly than the EU-Si sample.
\end{abstract}

\section{Introduction}

Much attention has been focused on the new materials in the use of biofibers as fillers and/or reinforcers elements in polymeric matrix which has been concerning on global ecological. Furthermore, the specific properties of this natural product such as low cost, light weight, renewable character, high specific strength and high modulus, reactive surface and the possibility to generate energy, without residue after burning at the end of their life-cycle, motivate their association with organic polymers to elaborate composite materials [1-4]. For the above reason, much work has been done in studying and developing general thermoplastic/natural fibers composites, especially wood plastic composites (WPCs). WPCs have successfully proven their applications in various fields such as lumber, decking and railing, window profiles, wall studs, door frames, furniture, pallets, fencing, docks, siding, architectural profiles, boat hulls, and automotive components $[4,5]$.

However, it is well known that different surface properties between the fiber and the matrix, i.e. the matrix is highly polar and hydrophilic while the filler is nonpolar and relatively hydrophobic, the compatibility and adhesion between matrix and filler are poor. Therefore, the coupling agents are much interested in order to improve thefibers /polymer compatibility and their interfacial adhesion $[4,6,7]$. It is known that coupling agents such as silane and olefin grafted maleic anhydride are an effective way to improve its dispersibility in

\footnotetext{
${ }^{\mathrm{a}}$ Corresponding author: siripan.m@en.rmutt.ac.th
}

polymer matrix as well, and hence ameliorate the polymer matrix, thus enhancing the properties of the resulting composites $[6,7]$.

The high density polyethylene (HDPE) is one of the most widely used in automotive, electric, and consumer applications and household applications. Beside, HDPE also enhances the comprehensive properties of semicrystalline engineering thermoplastic including a high rate of crystallization, good solvent resistance, thermal stability, and excellent processing properties and hence finds its applications in despite its exquisite properties; HDPE is very often modified with other polymers and particulate fillers [5].

Recently, much interest in new materials that can demonstrate WPCs products which developed polymeric matrix compatibility with wood flour for specific properties $[2,5]$. To improve the compatibility between hydrophobic (non-polar) thermoplastics and hydrophilic (polar) wood flours, the preparation of polymer composites with wood flour requires good compatibility in the interface between the polymer matrix and the wood flour. In this research we designed a wood plastic composite (WPCs) for household application such as furniture. The Eucalyptus wood residues (EWR) were used as a filled from these postconsumer materials in order to reduce the environmental impact and add value to these materials. Two type of coupling agents, Fusabond ${ }^{\mathrm{TM}}$ E-528 and Silane were used in this process in order to enhance compatibility and 
adhesion between wood-flours and thermoplastic matrices.

\section{Experiments}

\subsection{Materials}

High density polyethylene (HDPE H5818J) was purchased from SCG Plastic Co., Ltd. Fusabond ${ }^{\mathrm{TM}}$ E-528 (polyethylene-grafted maleic anhydride, PE-g-MA, MA) were supplied by Creative Polymer Co., Ltd. Amino Silane (Si) and Chiguard R326 (UV absorber) were purchased from Chitec Technology Co., Ltd. Eucalyptus fiber was from Bangkok Thailand. All samples were used as received. All other chemicals were used as supplied by the companies.

\subsection{Preparation of Eucalyptus wood-flour/HDPE}

Wood fibers were grinded using grinding machine. The Eucalyptus flours (EU) with particle size about $35 \mu \mathrm{m}$ was obtained from sieving method. The HDPE pellets and EU were dried at $80^{\circ} \mathrm{C}$ overnight prior used. The formulation of composites materials were presented in Table1. The $500 \mathrm{~g}$ of HDPE pellets was mixed with $5 \mathrm{wt} \%-20 \% \mathrm{wt}$ EU fine powder containing $1 \mathrm{wt} \%$ of coupling agent and $0.5 \mathrm{wt} \%$ of ChiguardR326. The glycerol 3 drop was added into the mixture. The EU was blended with HDPE using Twin Screw Extruder. The extruder barrel-temperatures zones were set at $190^{\circ} \mathrm{C}$. The screw speed was $60 \mathrm{rpm}$. The obtained EU/HDPE compound composites were cut into pellet size.

\subsection{Preparation of EU/HDPE slit.}

The EU/HDPE composite pellets were mixed using ThermoHakePolyDrive (single screw extruder). The barrel-temperatures zones were operated $190^{\circ} \mathrm{C}$. The screw speed was $40 \mathrm{rpm}$. The melting composite passes through the slit die (slit $=0.3 \mathrm{~mm} \times 4 \mathrm{~mm}$.) was first draw at $10 \mathrm{~m} . / \mathrm{min}$. and follow by recrystallized at $80^{\circ} \mathrm{C}$ and then second draw speed at $20 \mathrm{~m} . / \mathrm{min}$. The EU/HDPE slit was obtained.

Table 1. Formulation of composites materials.

\begin{tabular}{ccccccc}
\hline $\begin{array}{c}\text { Specimen } \\
\text { ID }\end{array}$ & Materials & Filler & $\begin{array}{c}\text { Coupling } \\
\text { agent }\end{array}$ & $\begin{array}{c}\text { HDPE } \\
(\% \mathrm{w} / \mathrm{w})\end{array}$ & $\begin{array}{c}\text { Eucalyptus } \\
(\% \mathrm{w} / \mathrm{w})\end{array}$ & $\begin{array}{c}\text { Coupling } \\
\text { agent }(\% \mathrm{w} / \mathrm{w})\end{array}$ \\
\hline EU-0 & HDPE & - & - & 100 & - & - \\
EU-MA 5 & HDPE & EU & PE-g-MA & 95 & 5 & 1 \\
EU-MA 10 & HDPE & EU & PE-g-MA & 90 & 10 & 1 \\
EU-MA 20 & HDPE & EU & PE-g-MA & 80 & 20 & 1 \\
EU-Si 5 & HDPE & EU & Silane & 95 & 5 & 1 \\
EU-Si 10 & HDPE & EU & Silane & 90 & 10 & 1 \\
EU-Si 20 & HDPE & EU & Silane & 80 & 20 & 1 \\
\hline \hline
\end{tabular}

\subsection{Thermal properties}

The melting temperature (Tm), crystalline temperature (Tc) and \%crystallinity of the HDPE/Eucalyptus fibers were explored using Differential Scanning Calorimetry. It was found that the melting temperature of the composites were obtained from heating cycle while the crystalline temperatures were obtained from cooling cycle. There 
were no significant difference of the melting temperature and crystalline temperature when increase the amount of Eucalyptus fibers from $0 \mathrm{wt} \%$ to $20 \mathrm{wt} \%$ as shown in Table 2. Melting temperature of the HDPE and their composites was around $131.2^{\circ} \mathrm{C}-133.6^{\circ} \mathrm{C}$. The \%crystallinity was slightly increased when increased the content of EU flour $5 \mathrm{wt} \%$ in both series samples. However, \%crystallinity slightly decreased at high content of EU flour 20wt\%. It was indicated that high content of EU flour effect the \%crystalline [5]. In addition, there was no significant effect of coupling agent on the melting temperature and crystalline temperature of composite.

Table 2. Thermal properties of HDPE and EU/HDPE composite with various content of EU from $5 \mathrm{wt} \%, 10 \mathrm{wt} \%$ and $20 \mathrm{wt} \%$ in the present of PE-g-MA or Silane.

\begin{tabular}{lccc}
\hline \hline Sample name & $\mathrm{Tm}\left({ }^{\circ} \mathrm{C}\right)$ & $\mathrm{Tc}\left({ }^{\circ} \mathrm{C}\right)$ & \%crystallinity \\
\hline \hline EU-0 & 131.2 & 115.4 & 56.86 \\
EU-MA 5 & 131.3 & 115.2 & 59.70 \\
EU-MA 10 & 131.6 & 115.1 & 57.11 \\
EU-MA 20 & 131.3 & 115.0 & 57.63 \\
EU-Si 5 & 131.8 & 116.2 & 63.58 \\
EU-Si 10 & 133.6 & 115.6 & 65.14 \\
EU-Si 20 & 131.5 & 115.6 & 56.52 \\
\hline \hline
\end{tabular}

\subsection{Scanning electron microscope (SEM)}

Figure 1 show the fracture surface of the composites slit with both of coupling agents at content of EU $10 \mathrm{wt} \%$. It was well known that the coupling agent could be effect on the interfacial properties of the composites [7]. The samples in which PE-g-MA and silane were used as coupling agents showed an improving in interfacial adhesion, observed for the considerable decreasing of gaps between the matrix and the dispersed phase as shown in Figure 1. This SEM image of EU-MA10 showed less holes and void spaces at the fracture surface, which implies that there was a good filler-matrix interaction between MA and the matrix. On the other hand, SEM image of EU-Si10 showed holes and void spaces at the fracture surface, which denotes that there was a poor filler-matrix interaction between silane and the matrix. This indicates that the PE-g-MA coupling agent was better than Si coupling agent. The presence of voids in the EU-Si series, as shown in Figure 1, may create stress concentration points, which in turn reduce the strength of the samples [6]. In addition, this would affect the mechanical and thermal performance of the substrate where the flours will potentially be used [6].

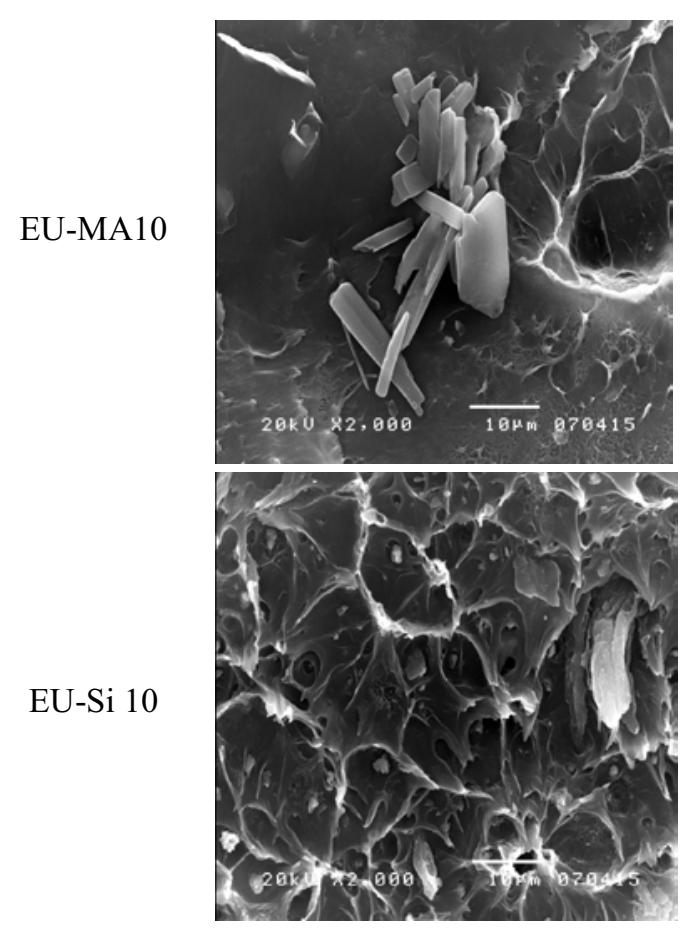

Figure 1. Fracture surface SEM images (2000x) of EU/MA and EU-Si10 composite with EU 10wt \% in the present of PE-g-MA and Silane.

\subsection{Tensile Test}

The mechanical properties of HDPE and EU/HDPE composite with various content of EU from $5 \mathrm{wt} \%$, $10 \mathrm{wt} \%$ and $20 \mathrm{wt} \%$ in the present of PE-g-MA and Silane were examined using Instron 5569 universal testing machine. The tensile strength and elongation of the composite were demonstrated in Figure 2 and Figure 3, respectively. The results showed that EU-MA5 had higher tensile strength more than EU-Si5. Moreover, tensile strength of the composite dramatically decreased when increased EU to $10 \mathrm{wt} \%$ but it was steady with increasing EU 20wt\%. Furthermore, tensile strength of EU-Si composite steadily decreased with increasing from $5 \mathrm{wt} \%$ to $20 \mathrm{wt} \%$ of EU in polymer composite. Regarding on SEM micrograph of EU-Si sample, it was noticed that the presence of voids of the EU-Si sample, as shown in Figure 1, may create stress concentration points, which in turn reduce the strength of the samples. Therefore, the mechanical properties of the EU-Si samples were poor than the EU-MA samples (Figure 2-3). It concluded that PE-g-MA coupling agent offer the better coupling agent over Silane coupling at higher content of EU flour.

The \%elongation was presented in Figure3. It was found that \%elongation was decreased with contents of EU in the composite. Moreover, \%elongation of EUMA samples slightly higher than EU-Si samples at all ratio. EU-MA with EU 5\%wt gave higher elongation. It was concluded that EU-MA5 was suitable to use due to its high enough tensile strength and high \%elongation. 


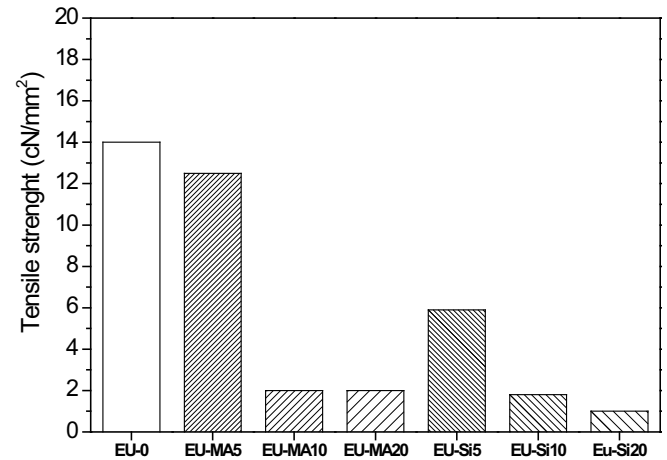

Figure 2. Tensile strength of HDPE and EU/HDPE composite with various content of EU from $5 \mathrm{wt} \%, 10 \mathrm{wt} \%$ and $20 \mathrm{wt} \%$ in the present of PE-g-MA and Silane.

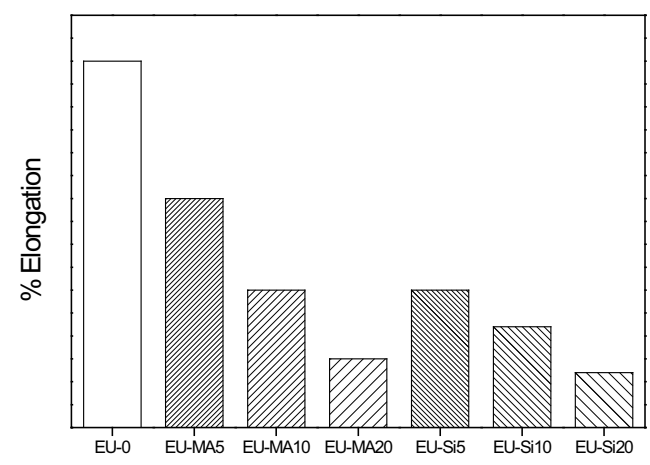

Figure 3. Elongation of HDPE and EU/HDPE composite with various content of EU from $5 \mathrm{wt} \%, 10 \mathrm{wt} \%$ and $20 \mathrm{wt} \%$ in the present of PE-g-MA and Silane.

\section{Conclusion}

The wood plastic composites (WPCs) have been successfully investigated. The effect of the coupling agents, PE-g-MA (MA) and Silane (Si) on the thermal properties, and mechanical properties of polymer composite were also studied. The Eucalyptus flour was blend with the HDPE at $5 \mathrm{wt} \%, 10 \mathrm{wt} \%$ and $20 \mathrm{wt} \%$. The EU/HDPE composite slits were obtained from single screw extruder. The results showed that both of coupling agents significantly affect the mechanical, structure and thermal properties of the EU/HDPE composite. In additionally, the high content of Eucalyptus flour may affect the \%crystalline of the polymer composite. The morphology of the EU/HDPE composite was also investigated by using SEM. It was noticed that the dispersion of fine Eucalyptus flour were observed with respected to the content of Eucalyptus flour. However, the PE-g-MA additive was presented the well mixing between Eucalyptus flour and polymer matrix.

\section{Acknowledgement}

I would like to thank Rajamangala University of Technology Thanyaburi for financial support.

\section{References}

1. D. G. Dikobe and A.S. Luyt, Express Polym. Lett., 4, 729 (2010)

2. Y. Lei and Q. Wu: Bioresour. Technol., 101, 3665 (2010)

3. V.J.R.R. Pita, E.E.M. Sampaio, and E.E.C. Monteiro, Polym. Test., 21, 545 (2002)

4. N. Saba, P.M. Tahir, and M. Jawaid, Polymers, 6, 2247 (2014)

5. F. Mengeloglu and A. Kabakci, Int. J. Mol. Sci., 9, 107 (2008)

6. Y. Xie, C.A.S. Hill, Z. Xiao, H. Militz, C. Mai, Composites: Part A, 41, 806 (2010)

7. A. L. Catto and R. Marlene, Journal. Mater.Sci.and Eng. b 3, 10, 641 (2013) 